DRAFT VERSION JUNE 23, 2021

Typeset using LATEX twocolumn style in AASTeX61

\title{
VLBA DETERMINATION OF THE DISTANCE TO NEARBY STAR-FORMING REGIONS. VIII. THE LKH $\alpha 101$ CLUSTER
}

\author{
Sergio A. Dzib, ${ }^{1}$ Gisela N. Ortiz-León,,${ }^{1,2}$ L. Loinard, ${ }^{3,4}$ A. J. Mioduszewski, ${ }^{5}$ L. F. RodríGuez, ${ }^{3}$ S.-N. X. Medina, ${ }^{1}$ And
} R. M. TORRES ${ }^{6}$

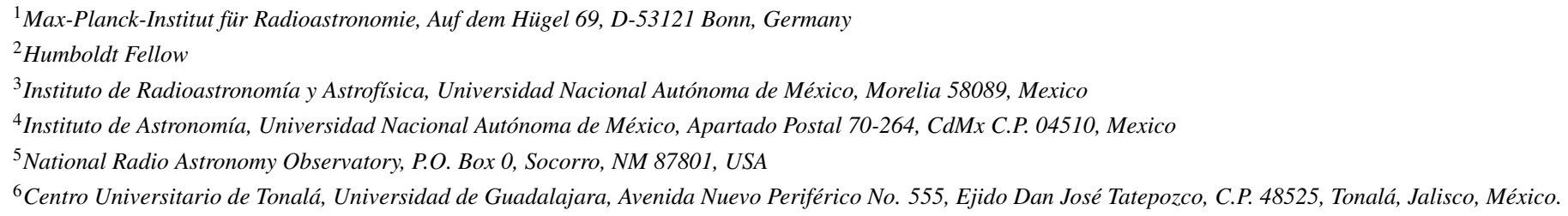

\section{ABSTRACT}

The $\mathrm{LkH} \alpha 101$ cluster takes its name from its more massive member, the $\mathrm{LkH} \alpha 101$ star, which is an $\sim 15 \mathrm{M}_{\odot}$ star whose true nature is still unknown. The distance to the $\mathrm{LkH} \alpha 101$ cluster has been controversial for the last few decades, with estimated values ranging from 160 to $800 \mathrm{pc}$. We have observed members and candidate members of the LkH $\alpha 101$ cluster with signs of magnetic activity, using the Very Long Baseline Array, in order to measure their trigonometric parallax and, thus, obtain a direct measurement of their distances. A young star member, LkH $\alpha 101$ VLA J043001.15+351724.6, was detected at four epochs as a single radio source. The best fit to its displacement on the plane of the sky yields a distance of $535 \pm 29 \mathrm{pc}$. We argue that this is the distance to the $\mathrm{LkH} \alpha 101$ cluster.

Keywords: astrometry — stars:formation — stars: individual (LKH $\alpha$ 101) — techniques: interferometric 


\section{INTRODUCTION}

$\mathrm{LkH} \alpha 101$ is a massive star, $M \sim 15 \mathrm{M}_{\odot}$, with an extinction of $A_{\mathrm{V}} \simeq 10$. It illuminates the reflection nebula NGC 1579 and has a directly imaged disk (see the review by Andrews \& Wolk 2008, and references therein). It also hosts a small HII region that is sustained by the ionized winds from its disk (Thum et al. 2013). $\mathrm{LkH} \alpha 101$ is associated with a cluster of young low-mass stars (hereafter, the $\mathrm{LkH} \alpha 101$ cluster), some of which are magnetically active (Becker \& White 1988; Stine \& O’Neal 1998; Osten \& Wolk 2009). These properties strongly suggest that $\mathrm{LkH} \alpha 101$ is a young high-mass star. However, there is an absence of stellar absorption features and, thus, there is no classification for its photosphere (Herbig et al. 2004). In fact, its spectroscopic properties have been compared to some post-main-sequence massive stars. As has been discussed by Andrews \& Wolk (2008), the true nature of $\mathrm{LkH} \alpha 101$ is still a mystery and this problem is compounded by its large distance uncertainty.

An extended discussion of the different suggested distances to the $\mathrm{LkH} \alpha 101$ cluster (which includes the $\mathrm{LkH} \alpha 101$ star) has been presented in the review by Andrews \& Wolk (2008) and we summarize it here. Initially, Herbig (1971) estimated a distance of 800 pc based on UBV photometry of two nearby early B-type stars. Later, Stine \& O’Neal (1998) suggested an entirely different value of $160 \mathrm{pc}$, arguing that the radio luminosities of $\mathrm{T}$ Tauri stars in the $\mathrm{LkH} \alpha 101$ cluster would be incompatible with that of $\mathrm{T}$ Tauri stars in Taurus-Aurigae if the cluster were at 800 pc. However, Herbig et al. (2004) noted that this method is inadvisable. Tuthill et al. (2002) favored a value of $d \simeq 340 \mathrm{pc}$, from model constraints on the star-disk mass for $\mathrm{LkH} \alpha 101$ and the proper motions of a companion. Herbig et al. (2004) obtained spectral parallax measurements to 40 young $\mathrm{LkH} \alpha 101$ cluster members with a wide range of spectral types and estimated $\mathrm{d} \simeq 700 \pm 200 \mathrm{pc}$. The conclusion of the discussion by Andrews \& Wolk (2008) was that most of the observational constrains suggest a distance between 500 pc to $700 \mathrm{pc}$. These authors also noted that the two different methods to identify cluster membership, by Feigelson \& Montmerle (1999) and Feigelson et al. (2005), are in good agreement when applied to the $\mathrm{LkH} \alpha 101$ cluster if its distance is about 550 pc. Clearly, the past suggested distances to the cluster have very large uncertainties because they are based only on interpretations of the properties of the stars. To date, there are no direct measurements of distances to any of the members of the $\mathrm{LkH} \alpha 101$ cluster and the most common assumed distance is $700 \mathrm{pc}$. An accurate distance to this region is fundamental to constrain the true nature of the $\mathrm{LkH} \alpha 101$ star.

Magnetically active young stars are excellent targets to measure trigonometric parallax and, thus, determine direct distances. They can be observed with the Very Long Baseline Interferometry (VLBI) technique (see Dzib et al. 2016;
Ortiz-León et al. 2017a,b; Kounkel et al. 2017, for recent results). By employing this technique, we observed suspected magnetically active young stars in the $\operatorname{LkH} \alpha 101$ cluster to measure their distance and provide a more accurate distance to the cluster.

\section{OBSERVATIONS AND DATA CALIBRATION}

We observed six young star members and a candidate member of the $\mathrm{LkH} \alpha 101$ cluster. These target sources are listed in Table 1. We used the Very Long Baseline Array (VLBA; Napier et al. 1994), operated by the Long Baseline Observatory (LBO), under projects BD165 and BD207. The observations used the multi-phase center capability provided by the VLBA DifX digital correlator (Deller et al. 2011). The observations of the first project were carried out on 2012 October 9 and October 11 , at a wavelength of $3.6 \mathrm{~cm}(\nu=8.42$ $\mathrm{GHz}$ ). In the first session (October 9), the first four targets in Table 1 were observed, while the other three were observed as part of the second session (October 11). Following the successful detections of three target sources, we initiated a series of multi-epoch observations (project BD207) at a wavelength of $6.0 \mathrm{~cm}(\nu=4.5 \mathrm{GHz})$ starting in 2017 March and, subsequently, we obtained a new observation every three months. The change of receiver was due to three reasons. First, the primary beam size is larger and we can cover all seven sources in a single pointing. Second, the new $6.0 \mathrm{~cm}$ band receiver is more sensitive than the $3.6 \mathrm{~cm}$ receiver. Finally, because of their negative spectral indices, the target sources are brighter at longer wavelengths. These last two reasons increase the SNR of the detection, increasing the precision of the position measurement.

Table 1. Observed sources. The names and infrared classes from Osten \& Wolk (2009).

\begin{tabular}{lcc}
\hline \hline Name & IR \\
$(\mathrm{LkH} \alpha 101 \mathrm{VLA})$ & Class & Detected? \\
\hline $\mathrm{J} 043010.87+351922.4$ & III & No \\
$\mathrm{J} 043016.04+351726.9$ & III & No \\
$\mathrm{J} 043017.90+351510.0^{\mathrm{a}}$ & $\ldots$ & Yes \\
$\mathrm{J} 043019.14+351745.6$ & II & Yes \\
$\mathrm{J} 042953.98+351848.2$ & III & No \\
J043001.15+351724.6 & III & Yes \\
J043002.64+351514.9 & II & No \\
\hline \hline & \\
\multicolumn{4}{c}{$a$ Candidate member. } \\
$b_{\text {This paper is based on the detections of this star. }}$
\end{tabular}

The observations of the targets were recorded as part of cycles with two minutes spent on-source and one minute spent on the main phase calibrator, J0429+3319. To improve the 
Table 2. Observation dates, synthesized beam size, and noise levels of the final maps around LkH $\alpha$ 101 VLA J043001.15+351724.6, as well as measured source position and flux densities.

\begin{tabular}{|c|c|c|c|c|c|c|c|c|}
\hline $\begin{array}{l}\text { Mean UT date } \\
\text { (yyyy.mm.dd/hh:mm) }\end{array}$ & Julian Day & $\begin{array}{l}\text { Synthesized beam } \\
\left(\theta_{\text {maj }} \times \theta_{\text {min }} ; \text { P.A. }\right)\end{array}$ & $\begin{array}{c}\sigma_{\text {noise }} \\
\left(\mu \mathrm{Jybm}^{-1}\right)\end{array}$ & $\begin{array}{c}\alpha(\mathrm{J} 2000.0) \\
04^{\mathrm{h}} 30^{\mathrm{m}} \\
\end{array}$ & $\sigma_{\alpha}$ & $\begin{array}{c}\delta(\mathrm{J} 2000.0) \\
35^{\circ} 17^{\prime} \\
\end{array}$ & $\sigma_{\delta}$ & $\begin{array}{c}f_{\nu} \\
(\mathrm{mJy})\end{array}$ \\
\hline 2012.10.09/10:17 & 2456211.93 & $0^{\prime \prime} .0021 \times 0^{\prime \prime} .0008 ; 6.5^{\circ}$ & 36 & 1.146281 & 0.000006 & 24.’43957 & 0.’00014 & $0.26 \pm 0.04$ \\
\hline $2017.03 .25 / 21: 46$ & 2457838.41 & $0^{\prime \prime} .0036 \times 0^{\prime \prime} .0012 ; 18.6^{\circ}$ & 23 & & 0.000008 & 24:"41308 & 0.’00017 & $0.20 \pm 0.02$ \\
\hline 2017.06.17/16:16 & 2457922.18 & $0^{\prime \prime} .0034 \times 0^{\prime \prime} .0012 ; 15.4^{\circ}$ & 21 & 1.146934 & 0.000007 & 24:"41223 & 0.’00022 & $0.16 \pm 0.02$ \\
\hline 2017.09.11/10:38 & 2458007.94 & $0^{\prime \prime} .0046 \times 0^{\prime \prime} .0015 ;-1.0^{\circ}$ & 27 & 1.147056 & 0.000002 & 24.”41143 & 0.’00007 & $0.81 \pm 0.05$ \\
\hline
\end{tabular}
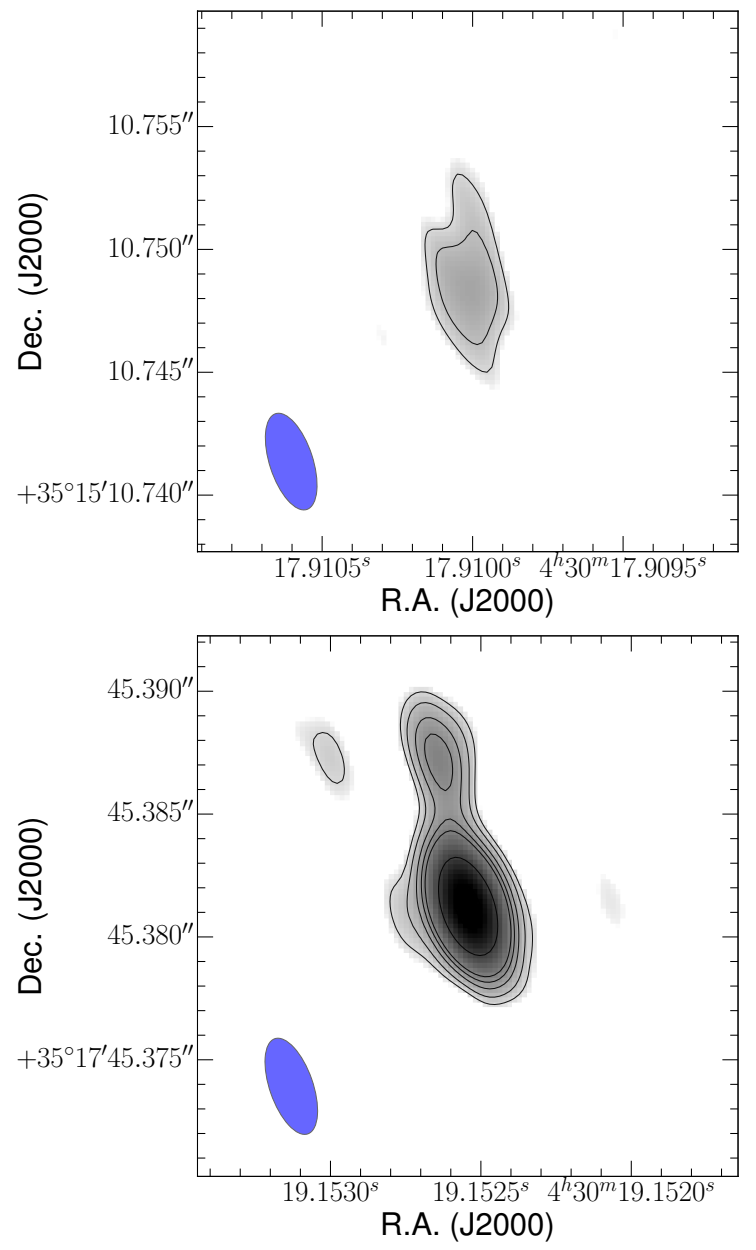

Figure 1. $\operatorname{LkH} \alpha$ 101 VLA J043017.90+351510.0 (top) and $\mathrm{LkH} \alpha 101$ VLA J043019.14+351745.6 (bottom) as detected on 2017 March 25. The noise levels are $31 \mu \mathrm{Jy}^{\text {beam }^{-1}}$ and $38 \mu \mathrm{Jy}$ beam $^{-1}$ for the top and bottom images, respectively. The contour levels are $-3,4,6,9,12,15,30$, and 50 times the noise level. The size of the synthesized primary beam for both images is 4.1 mas $\times$ 1.6 mas; P.A. $=18.5$, and is displayed as a filled blue ellipse in the bottom left corner of each image.

quality of the phase calibration, we also observed every 30 minutes the secondary calibrators J0443+3441, J0414+3418, and J0418+3801. Additionally, about two dozen ICRF quasars distributed over the entire visible sky were observed during the observations (conforming the so-called geodetic blocks); those are used to improve tropospheric calibration (e.g., Reid \& Brunthaler 2004). These geodetic blocks were observed at the beginning and at the end of each epoch. The observation lengths of each epoch were 3.0 and 2.5 hours for the projects BD165 and BD207, respectively.

The data were edited and calibrated using the Astronomical Image Processing System (AIPS; Greisen 2003). The basic data reduction followed the standard VLBA procedure for phase-referenced observations, including the multicalibrator schemes ${ }^{1}$ and the tropospheric and clock corrections obtained from the geodetic blocks (see Loinard et al. 2007; Torres et al. 2007; Dzib et al. 2010, for a detailed description of these calibration steps). After calibration, the visibilities were first imaged with a pixel size of $100 \mu$ as using a natural weighting scheme (ROBUST $=5$ in AIPS) and covering an area of $\sim 1$ square arcsecond. As this scheme provides the best possible noise level, we used these images to search for source detections. When a detection was obtained, we constructed new images, around the source, with a weighting scheme intermediate between natural and uniform (ROBUST $=0$ ) using a pixel size of $50 \mu$ as. In these last images we lost some sensitivity, but gained some angular resolution, enabling a slightly better determination of the source positions at each epoch. These images were then also corrected for the response of the primary beam. The r.m.s. noise levels, $\sigma_{\text {noise }}$, in the final images were $21-36 \mu \mathrm{Jy}$ beam $^{-1}$. The parameters of the images obtained at individual epochs are given in Table 2. From these images, the source position, flux, and deconvolved size were determined by using a two-dimensional fitting procedure (task JMFIT in AIPS).

\section{RESULTS}

Three of the target sources were detected in our observations (see Figures 1 and 2, and Table 1). LkH $\alpha 101$ VLA 043001.15+351724.6 and LkH $\alpha 101$ VLA 043017.90+351510.0

\footnotetext{
${ }^{1}$ The phase transfer from the main calibrator to the secondary calibrator J0414+3418 did not work properly, so the latter source was excluded from the multi-calibrator correction.
} 

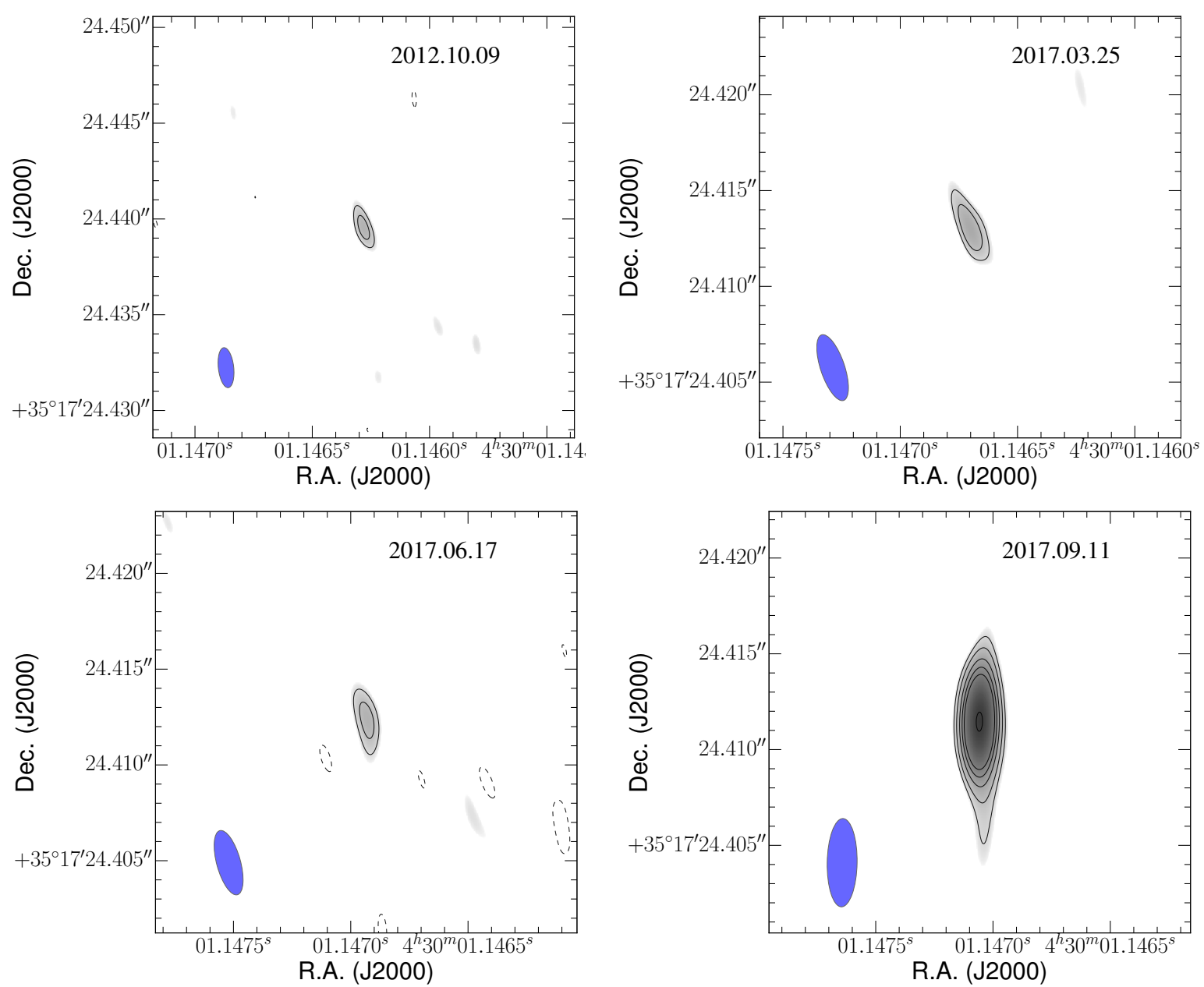

Figure 2. $\mathrm{LkH} \alpha 101$ VLA J043001.15+351724.6 as detected in each epoch. The contour levels are as in Figure 1. The noise levels and the size of the synthesized primary beams are listed in Table 2. The latter are displayed as filled blue ellipses in the bottom left corner of each image.

were detected as single compact radio sources in all four epochs. $\operatorname{LkH} \alpha 101$ VLA 043019.15+351745.6, on the contrary, had a more complex morphology. In the first epoch a single source was detected, however in the last three epochs we detected two sources in each image (e.g., bottom of Figure 1). This multi-epoch detection of two radio sources indicates that $\mathrm{LkH} \alpha$ 101 VLA J043019.14+351745.6 is a possible tight binary system, and it will be interesting for further study.

The position of the source related to $\mathrm{LkH} \alpha 101 \mathrm{VLA}$ $043017.90+351510.0$ did not significantly change between the different epochs suggesting that it is a background object and not a member of the $\mathrm{LkH} \alpha 101$ cluster. The complexity of LkH $\alpha 101$ VLA J043019.14+351745.6 and the low number of detections make it difficult to perform an accurate astrometric analysis. This system will be further analyzed in a future paper when more observations are collected. The young star LkH $\alpha$ 101 VLA 043001.15+351724.4 was well detected in four epochs and we will focus our astrometric analysis on it. In the three first detected epochs, JMFIT can- not deconvolve LkHa 101 VLA J043001.15+351724.6 to a finite size. The fourth epoch is also consistent with a point source, although it would also be consistent with a deconvolved size up to $0 .^{\prime \prime} 0022 \times 0 . .^{\prime \prime} 0004$; P.A. $=33^{\circ}$. Thus the target at this epoch might be marginally resolved (on account of the unresolved nature of the source at the other epochs, we consider that it is more likely that the data contain remaining phase errors). The images of its radio emission at all epochs are shown in Figure 2.

\subsection{Astrometry}

The displacement of LkH $\alpha$ 101 VLA 043001.15+351724.4 on the plane of the sky can be modeled as a combination of a trigonometric parallax $(\varpi)$ and linear proper motions $(\mu)(\mathrm{e} . \mathrm{g}$. Loinard et al. 2007). The fluxes and measured equatorial positions are presented in Table 2. The barycentric coordinates of the Earth appropriate for each observation were calculated using the NOVAS routines distributed by the US Naval Observatory. The reference epoch was taken at JD 2457108.94 $\equiv \mathrm{J} 2015.24$, the mean epoch of the observations. The best 


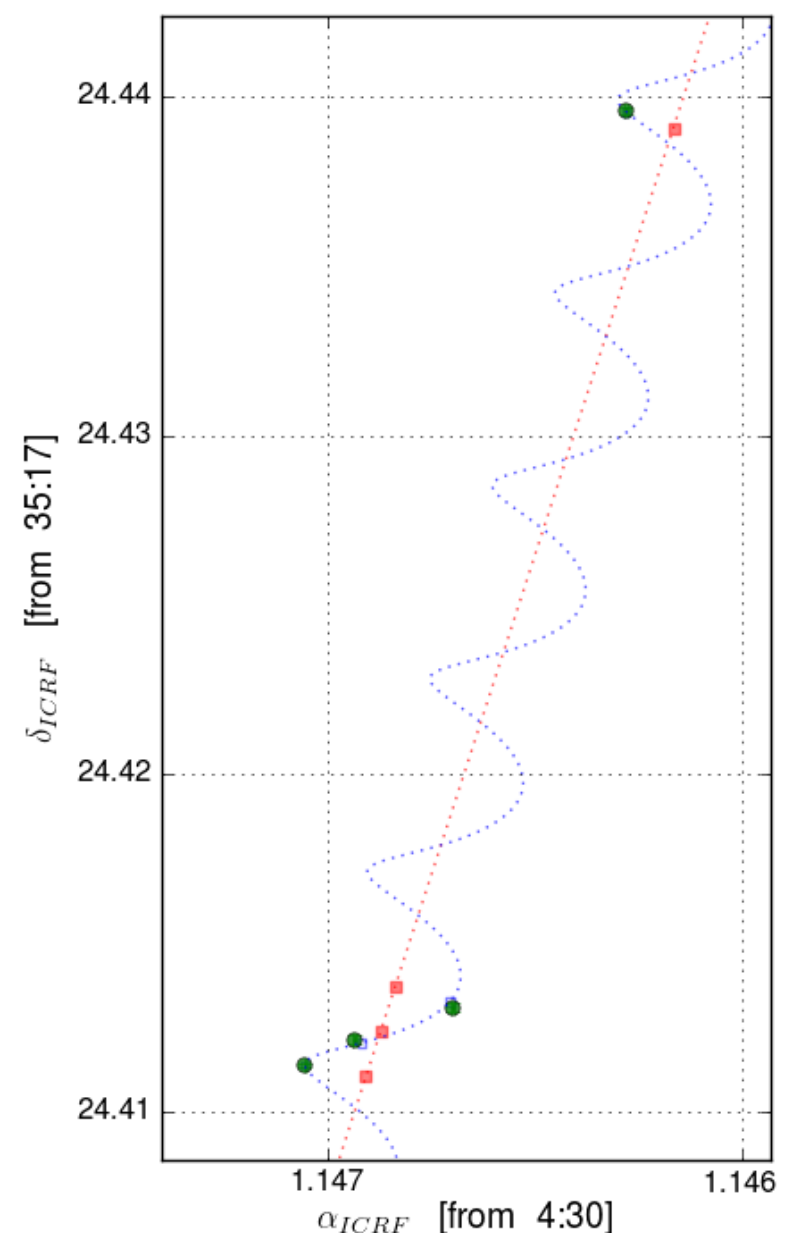

Figure 3. Measured positions (green circles) and best fit to the movement (dotted blue line) of $\mathrm{LkH} \alpha$ 101 VLA J043001.15+351724.6. Blue squares indicate the expected position from the best fit model. The red dotted line is the model after subtracting the reflex movement of the trigonometric parallax. The red squares indicate the position of the source at the observed epochs expected from the model after correction for reflex parallax motion.

fit to the data assuming a uniform proper motion (Figure 3) yields the following astrometric elements:

$$
\begin{aligned}
\alpha_{J 2015.24} & =04^{\mathrm{h}} 30^{\mathrm{m}} 01.146538 \pm 0.000009 \\
\delta_{J 2015.24} & =35^{\circ} 17^{\prime} 24^{\prime \prime} .4251 \pm 0^{\prime \prime} .0001 \\
\mu_{\alpha} \cos \delta & =1.86 \pm 0.04{\text { mas } \mathrm{yr}^{-1}}^{\mathrm{s}}+0.05{\text { mas } \mathrm{yr}^{-1}} \\
\mu_{\delta} & =-5.70 \pm 0.10 \text { mas. }
\end{aligned}
$$

This parallax corresponds to a distance of $d=535 \pm 29 \mathrm{pc}$. The post-fit r.m.s. values are 0.15 and 0.16 mas in right ascension and declination, respectively. Systematic errors of 0.10 and 0.13 mas (in right ascension and declination, respectively), were added in quadrature to the uncertainties delivered by JMFIT to obtain a reduced $\chi^{2}=1$.

\section{DISCUSSION AND CONCLUSION}

The measurement of a trigonometric parallax is independent of any assumption of the properties of the star, since it is a purely geometric method. Consequently, this is a direct determination of distances.

The young star LkH $\alpha$ 101 VLA J043001.15+351724.6 is a bona fide member of the $\mathrm{LkH} \alpha 101$ cluster (Herbig et al. 2004; Osten \& Wolk 2009). Therefore, its distance gives us an accurate approach on the distance to this cluster. The angular size of the cluster is $\sim 8^{\prime}$, corresponding to a physical size of $1.25 \mathrm{pc}$ at the distance of $535 \mathrm{pc}$. Because this size is much smaller than our distance error we can safely assume that the distance to the cluster, including the $\mathrm{LkH} \alpha 101$ star, its most massive member, is also $535 \pm 29$ pc. This result confirms the suggestion by Andrews \& Wolk (2008) that the distance to the $\mathrm{LkH} \alpha 101$ cluster ranges between 500 to 700 $\mathrm{pc}$, and whom also favored a distance of $550 \mathrm{pc}$. Given this range of values, our result has reduced the uncertainty on the distance to the $\mathrm{LkH} \alpha 101$ cluster by a factor of three. Considering that this is the first direct measurement of a distance to one of the star members of the $\mathrm{LkH} \alpha 101$ cluster, our result is also the most well founded distance to the cluster until now.

G.-N.O.L acknowledges support from the Alexander von Humboldt Foundation in the form of a Humboldt Fellowship. L.L. and L.R. acknowledges the financial support of DGAPA, UNAM (project IN112417), and CONACyT, México. S.-N.X.M. acknowledges IMPRS for a Ph.D. research scholarship. The Long Baseline Observatory is a facility of the National Science Foundation operated under cooperative agreement by Associated Universities, Inc.

Software: AIPS (Greisen 2003).

\section{REFERENCES}


Deller, A. T., Brisken, W. F., Phillips, C. J., et al. 2011, PASP, 123, 275

Dzib, S., Loinard, L., Mioduszewski, A. J., et al. 2010, ApJ, 718, 610

Dzib, S. A., Ortiz-León, G. N., Loinard, L., et al. 2016, ApJ, 826, 201

Feigelson, E. D., Getman, K., Townsley, L., et al. 2005, ApJS, 160, 379

Feigelson, E. D. \& Montmerle, T. 1999, ARA\&A, 37, 363

Greisen, E. W. 2003, Information Handling in Astronomy Historical Vistas, 285, 109

Herbig, G. H. 1971, ApJ, 169, 537

Herbig, G. H., Andrews, S. M., \& Dahm, S. E. 2004, AJ, 128, 1233

Kounkel, M., Hartmann, L., Loinard, L., et al. 2017, ApJ, 834, 142

Loinard, L., Torres, R. M., Mioduszewski, A. J., et al. 2007, ApJ, 671,546
Napier, P. J., Bagri, D. S., Clark, B. G., et al. 1994, IEEE Proceedings, 82, 658

Ortiz-León, G. N., Dzib, S. A., Kounkel, M. A., et al. 2017a, ApJ, 834, 143

Ortiz-León, G. N., Loinard, L., Kounkel, M. A., et al. 2017b, ApJ, 834, 141

Osten, R. A. \& Wolk, S. J. 2009, ApJ, 691, 1128

Reid, M. J. \& Brunthaler, A. 2004, ApJ, 616, 872

Stine, P. C. \& O’Neal, D. 1998, AJ, 116, 890

Thum, C., Neri, R., Báez-Rubio, A., \& Krips, M. 2013, A\&A, 556, A129

Torres, R. M., Loinard, L., Mioduszewski, A. J., \& Rodríguez, L. F. 2007, ApJ, 671, 1813

Tuthill, P. G., Monnier, J. D., Danchi, W. C., Hale, D. D. S., \& Townes, C. H. 2002, ApJ, 577, 826 